\title{
Chronic Toxoplasmosis Modulates the Induction of Contact Hypersensitivity by TNCB in Mouse Model
}

\author{
Zhaoshou Yang, Hye-Jin Ahn, Ho-Woo Nam* \\ Department of Parasitology, College of Medicine, The Catholic University of Korea, Seoul 06591, Korea
}

\begin{abstract}
Mouse models of chronic toxoplasmosis and atopic dermatitis (AD) were combined to clarify the effect of opportunistic Toxoplasma gondii infection on the development of $A D$. AD was induced as a chronic contact hypersensitivity (CHS) with repeated challenge of 2,4,6-trinitro-1-chlorobenzene (TNCB) on the dorsal skin of mice. TNCB induced skin thickness increases in both normal and toxoplasmic mice. The changing patterns were different from the sigmoidal which saturated at 20 days in normal mice to the convex saturated at 12 days in toxoplasmic mice with the crossing at 18 days. Compared to normal mice, toxoplasmic mice presented CHS more severely in earlier times and then moderately in later times. These data suggest that host immune modification by $T$. gondii infection enhances $\mathrm{CHS}$ in early times of atopic stimulation but soothes the reaction of $\mathrm{CHS}$ in later times in mouse model.
\end{abstract}

Key words: Toxoplasma gondii, toxoplasmosis, contact hypersensitivity, atopic dermatitis, animal model

Toxoplasma gondii infects humans and animals chronically all over the world [1]. Although T. gondii modulates innate and adaptive immune responses of the host [2,3], the effects of $T$. gondii infection on autoimmune diseases in humans and animal models such as atopic dermatitis are not well known. The chronic contact hypersensitivity (CHS) induced by repeated epicutaneous exposure to hapten is one of animal models with atopic dermatitis-like skin lesions [4]. To this end, we initiated the experiment that challenging BALB/c mice with TNCB treatment [4] after infection of T. gondii type II Me49 strain chronically [5]. To gain insight into the changing of CHS in infected mice, we designed the trail to evaluate the hall marker of atopic dermatitis, the dorsal swelling and clinical skin severity.

$\mathrm{BALB} / \mathrm{c}$ mice were housed at $22^{\circ} \mathrm{C}$ with a 12 -hr light-dark cycle in the semi-specific pathogen free (SPF) area in accordance with a protocol approved by the Animal Care and Use Committee of Catholic University of Korea. A tissue cyst forming strain of T. gondii, Me49, is passaged in BALB/c mice every 3 months per oral route in a dose of 30 cysts/mouse. Five female mice in both groups were anesthetized, and the hair of

\footnotetext{
- Received 15 September 2015, revised 21 October 2015, accepted 1 November 2015. *Corresponding author (howoo@catholic.ac.kr) (C) 2015, Korean Society for Parasitology and Tropical Medicine This is an Open Access article distributed under the terms of the Creative Commons Attribution Non-Commercial License (http://creativecommons.org/licenses/by-nc/3.0) which permits unrestricted non-commercial use, distribution, and reproduction in any medium, provided the original work is properly cited.
}

their dorsal skin was shaved weekly using an electric shaver, and then were sensitized with 5\% TNCB (Sigma Aldrich, St. Louis, Missouri, USA) dissolved in acetone: olive oil (4:1) at $50 \mu \mathrm{l} /$ dorsal skin. A week later (designated as day 0 ), for the elicitation of allergic contact dermatitis, mice were applied repeatedly with $1 \% \mathrm{TNCB}$ at $50 \mu \mathrm{l} /$ dorsal skin up to 28 days at 2-3 day intervals [6]. The thickness of dorsal skins was measured by a dial caliper gauge (Ozaki Seisakusho Co., Tokyo, Japan). All measurements were done right before the treatment of the drug. Dorsal skin swelling $(\mathrm{mm})=$ thickness of dorsal skin on day n-thickness of dorsal skin before the first treatment of TNCB in each mouse.

The statistical analysis of the differences in the means for each experimental group of mice was carried out using the Student's $t$-tests. All values are expressed as means \pm SD. $P$-values less than 0.05 were considered statistically significant.

With repeated exposure to TNCB, the dorsal skin swelling in T. gondii-infected group was increased rapidly (Fig. 1) and peaked on day 12 to be retained up to day 28 as a convex mode, while in normal mice the swelling followed a sigmoidal mode which peaked on day 20. Two patterns were crossed at approximately day 18 such that the dorsal skin swelling was thicker in T. gondii-infected group up to day 18 and afterwards in normal group reversely (Fig. 1A). On day 12, the skin swelling of T. gondii-infected mice was obviously different from that of normal mice and on day 20, reversed significantly (Fig. 1B). The skin features showed visually a marked reduction in sever- 
A

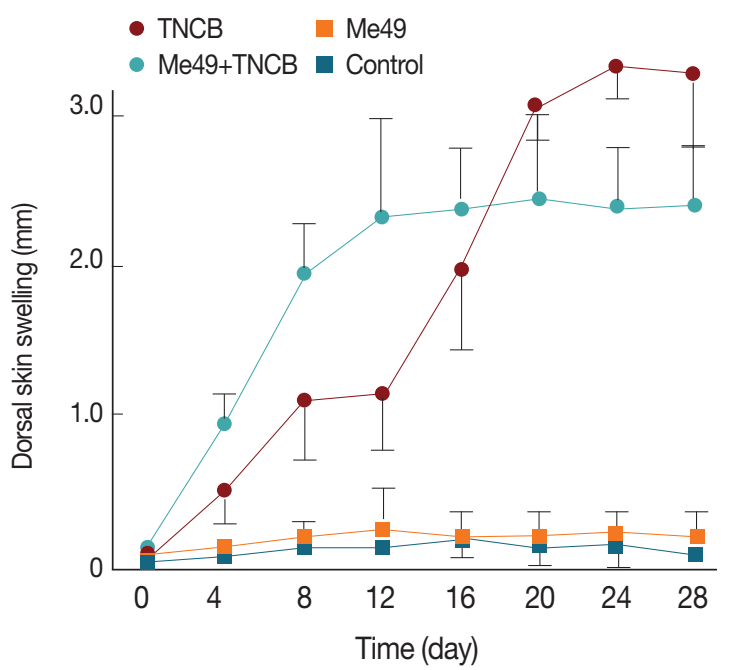

$\mathrm{B}$

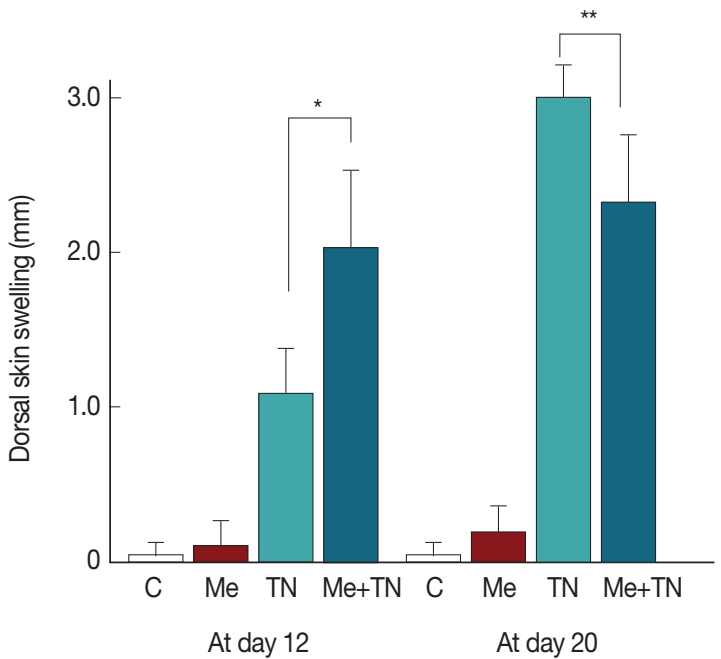

Fig. 1. (A) Time courses of dorsal skin swelling of mice challenged with TNCB. Parameters were obtained right before the treatment of the drug. Five normal mice (Control) and 5 T. gondii Me49 strain infected mice (Me49) were challenged with acetone: olive oil (4:1). Five normal mice (TNCB) and 5 Me49 infected mice (Me49+TNCB) were challenged with TNCB. (B) The difference of the dorsal skin swelling between normal and $T$. gondii-infected mice is significant on day 12 and on day $20\left({ }^{*}\right.$ and $\left.{ }^{* \star} P<0.05\right)$.
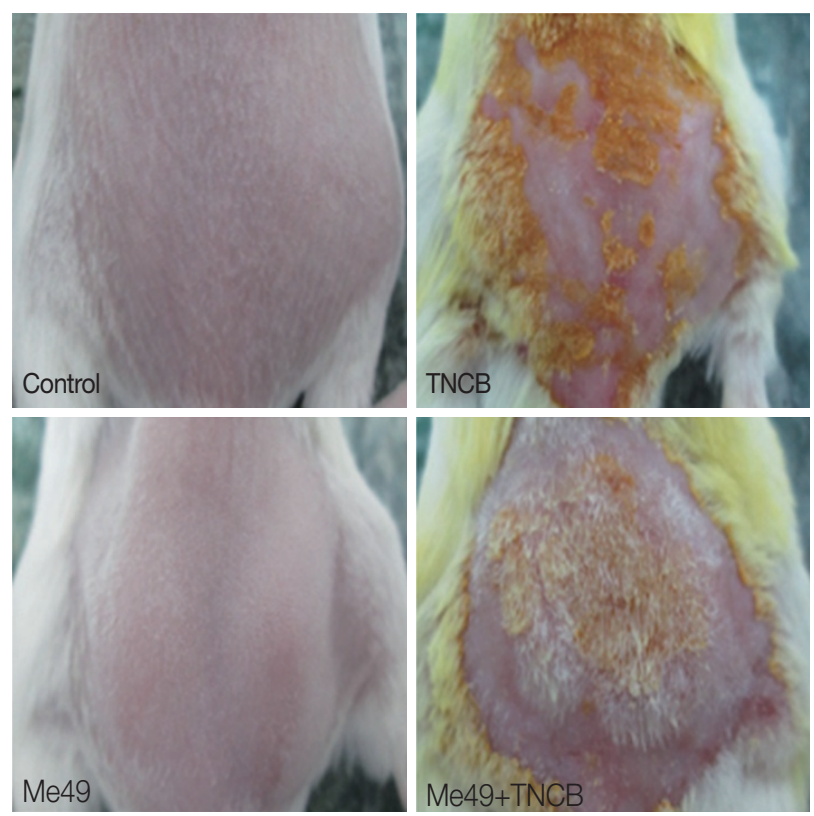

Fig. 2. Effect of TNCB treatment on the dorsal skin in mice on day 12. The clinical severity of dermatitis on TNCB and Me49+TNCB is significantly different on day 12. Mouse in Me49+TNCB was undergoing hair-growth on day 12, while mouse in TNCB was undergoing severe atopic dermatitis-like skin lesions.

ity of dermatitis and more rapid hair re-growth in T. gondii-infected mice. Control mice mostly exhibited severe symptoms of dermatitis including hemorrhage, edema, excoriation/erosion, and dynes/scaling, while T. gondii-infected mice presented with mild or moderate clinical skin severity (Fig. 2). These data provided direct evidence of the effects of $T$. gondii infection on CHS in mouse model.

These differences may derive from the immune response of mice infected with T. gondii and CHS mice model induced by TNCB. At the acute phase of Me49 infection, T-helper (Th) 1-type immune response is dominantly activated [2]. The activation of $\mathrm{CD} 4^{+} \mathrm{T}$ cell can enhance the inflammation and dissemination of $T$. gondii in mouse body including the brain [7]. At the acute phase of CHS induced by TNCB, Th1-type immune response is dominantly activated. Meanwhile, T. gondii induce the activation of signal transducer and activator of transcription 6 (STAT6) [8], which play a critical role in the induction phase of CHS [9]. From acute phase to chronic phase of Me49 infection, the immune responses of host shift from Th1type to Th2-type dominant response accompanying with stronger $\mathrm{CD}^{+} \mathrm{T}$ cell responses [2]. In addition, $\mathrm{CD}^{+} \mathrm{T}$ cell responses control the dissemination of T. gondii. Accompanying with the other responses of host immunity, T. gondii is transformed from fast growing tachyzoites to slowly growing bradyzoites and eventually forms tissue cysts [2]. Meanwhile, T cell function was impaired by T. gondii infection [3]. At the acute phase of CHS induced by TNCB, Th1-type immune response is dominantly activated [4]. While at the chronic phase of CHS induced by TNCB, the immune responses of host shift from Th1-type to a Th2-type response.

In the present study, mice were in the chronic phase of Me49 infection. At the acute phase of CHS induced by TNCB, 
the stronger $\mathrm{CD}^{+} \mathrm{T}$ cell activation, Th1-type response, enhances the inflammation by $T$. gondii. The immune responses of T. gondii inflammation [10], such as IL-4 independent activation of STAT6, contribute to the steep increment of dorsal skin under which there are plenty of lymph organs preferred to be infected by T. gondii. At chronic phase of CHS induced by $\mathrm{TNCB}$, the immune responses at the chronic phase of Me49 infection, in particular $\mathrm{CD} 8^{+} \mathrm{T}$ cell activation and impairment of $\mathrm{T}$ cell function may have an immunosuppressive effect on the clinical severity of AD-like skin lesions.

Taken together, the preliminary data has showed BALB/C mice in the chronic phase of Me49 infection are sensitive to TNCB biphasically but resistant to AD-like skin lesions induced by TNCB. These results will hopefully facilitate the understanding of the effect of autoimmune diseases on those $T$. gondii-infected population.

\section{ACKNOWLEDGMENT}

This research was supported in part by the Basic Science Research Program through the National Research Foundation of Korea (NRF) funded by the Ministry of Education, Science and Technology (no. NRF-2012R1A1A 2002612).

\section{REFERENCES}

1. Yang Z, Cho PY, Ahn SK, Ahn HJ, Kim TS, Chong CK, Hong SJ, Cha $\mathrm{SH}, \mathrm{Nam} \mathrm{HW}$. A surge in the seroprevalence of toxoplas- mosis among the residents of islands in Gangwha-gun, Incheon, Korea. Korean J Parasitol 2012; 50: 191-197.

2. Hunter CA, Sibley LD. Modulation of innate immunity by Toxoplasma gondii virulence effectors. Nat Rev Microbiol 2012; 10: 766-778.

3. Rodrigues V, Cordeiro-da-Silva A, Laforge M, Ouaissi A, Akharid $\mathrm{K}$, Silvestre R, Estaquier J. Impairment of T cell function in parasitic infections. PLoS Negl Trop Dis 2014; 8: e2567.

4. Harada D, Takada C, Tsukumo Y, Takaba K, Manabe H. Analyses of a mouse model of the dermatitis caused by 2,4,6-trinitro1-chlorobenzene (TNCB)-repeated application. J Dermatol Sci 2005; 37: 159-167.

5. Subauste C. Animal models for Toxoplasma gondii infection. Curr Protoc Immunol 2012; Chapter 19: Unit 19.3. 1-23.

6. Matsumoto K, Mizukoshi K, Oyobikawa M, Ohshima H, Tagami H. Establishment of an atopic dermatitis-like skin model in a hairless mouse by repeated elicitation of contact hypersensitivity that enables to conduct functional analyses of the stratum corneum with various non-invasive biophysical instruments. Skin Res Technol 2004; 10: 122-129.

7. Kamerkar S, Davis PH. Toxoplasma on the brain: understanding host-pathogen interactions in chronic CNS infection. J Parasitol Res 2012; 2012: 589295.

8. Ahn HJ, Kim JY, Nam HW. IL-4 independent nuclear translocalization of STAT6 in HeLa cells by entry of Toxoplasma gondii. Korean J Parasitol 2009; 47: 117-124.

9. Yokozeki H, Ghoreishi M, Takagawa S, Takayama K, Satoh T, Katayama I, Takeda K, Akira S, Nishioka K. Signal transducer and activator of transcription 6 is essential in the induction of contact hypersensitivity. J Exp Med 2000; 191: 995-1004.

10. Melo MB, Jensen KD, Saeij JP. Toxoplasma gondii effectors are master regulators of the inflammatory response. Trends Parasitol 2011; 27: 487-495. 
